\title{
Prevalence and risk factors for Plasmodium falciparum malaria in pregnant women attending antenatal clinic in Bobo-Dioulasso (Burkina Faso)
}

\author{
Mamoudou Cisse ${ }^{1,3^{*}}$, Ibrahim Sangare ${ }^{2,3}$, Guekoun Lougue ${ }^{1}$, Sanata Bamba ${ }^{2,3}$, Dramane Bayane ${ }^{4}$ \\ and Robert Tinga Guiguemde ${ }^{1,3}$
}

\begin{abstract}
Background: Malaria during pregnancy remains a serious public health problem. The aim of this study was to determine the prevalence and possible risk factors for malaria in pregnant women attending antenatal clinic at two primary health facilities in Bobo-Dioulasso.

Methods: We conducted a cross sectional study from September to December 2010 in two primary health facilities located in the periurban area of Bobo-Dioulasso. Pregnant women attending antenatal clinic (ANC) were included in the study after signing informed consent. For each participant, the social-demographic profile, malaria and obstetric histories were investigated through a questionnaire. Peripheral blood was collected and thick and thin blood smears were prepared to check Plasmodium falciparum parasitaemia. Hemoglobin concentration was measured. The associations between age, parity, gestational age, schooling, number of ANC visits, use of IPTp-SP, use of insecticide-treated nets (ITN) and anemia with the occurrence of $P$. falciparum malaria infection during pregnancy were analyzed through logistic regression.
\end{abstract}

Results: During the period of study, 105 (18.1\%) out of 579 pregnant women were infected by $P$. falciparum. The hemoglobin concentration mean was $10.5 \pm 1.7 / \mathrm{dL}$ and was significantly lower in pregnant women with malaria infection $(9.8 \mathrm{~g} / \mathrm{dL} \pm 1.6)$ than in those who had no malaria infection $(10.6 \mathrm{~g} / \mathrm{dL} \pm 1.7)(P<0.001)$. Multivariate analysis indicated that, education (AOR 1.9, 95\% Cl=[1.2-3.2]), parity [primigravidae (AOR 5.0, 95\% Cl=[2.5-9.8]) and secundigravidae (AOR 2.1, 95\% Cl=[1.2-3.8])], and anaemia (AOR 2.1,95\% Cl=[1.3-3.5]) were significantly associated with $P$. falciparum malaria infection. The use of IPTp-SP was not associated with P. falciparum malaria infection.

Conclusions: $P$. falciparum malaria infection is common in pregnant women attending antenatal clinic and anaemia is an important complication. The results show that the use of IPTP-SP does not reduce the risk of malaria incidence during pregnancy.

Keywords: Malaria, Prevalence, Pregnancy, Risk factors, Burkina Faso

\section{Background}

Malaria during pregnancy is a serious public health problem in Sub-Saharan Africa. It is estimated that each year, approximately 25 million pregnant women in Sub-Saharan Africa are at risk of Plasmodium falciparum malaria infection during pregnancy [1]. Malaria during

\footnotetext{
* Correspondence: cisse_m@yahoo.fr

${ }^{1}$ Centre MURAZ, Bobo-Dioulasso, Burkina Faso

${ }^{3}$ Institut Supérieur des sciences de la Santé, Université Polytechnique de Bobo-Dioulasso, Bobo-Dioulasso, Burkina Faso

Full list of author information is available at the end of the article
}

pregnancy leads to serious adverse effects on the mother and the child. Indeed although malaria during pregnancy might be asymptomatic due to high level of acquired immunity in mothers residing in high transmission areas, it is still associated with maternal anaemia, abortion, prematurity and low birth weight $[1,2]$. Moreover, severe maternal anaemia increases the mother's risk of death and malaria related anaemia is estimated to cause as many as 10,000 maternal death each year in Africa [3]. In Burkina Faso, 22,130 cases of severe malaria were recorded during pregnancy in 2011 resulting in 97 maternal deaths [4]. 
To face this flail, the World Health Organization (WHO) adopted the intermittent preventive treatment with sulfadoxine-pyrimethamine during pregnancy (IPTpSP) [3]. This policy has been adopted by the Burkina Faso ministry of health in 2005. Several epidemiological studies have been conducted in pregnancy before and after the implementation of IPTp-SP in Burkina Faso [5-17]. The prevalence of maternal peripheral $P$. falciparum infection assessed by microscopy was $24 \%$ in urban area [12] and varied from $19.4 \%$ to $50.8 \%$ in rural area $[5-9,13]$.

Factors influencing malaria prevalence in pregnant women include maternal age, parity, use of prophylaxis, nutrition, host and parasite genetics and level of antiparasitic immunity [18] with conflicting data concerning maternal age and parity depending on urban or rural setting [19-23]. So far, only one study had investigated the risk factors of malaria during pregnancy in Burkina Faso [5]. This study had been carried out in a rural area and one year after the implemention of IPTp-SP pilot program.

Understanding the epidemiology of malaria during pregnancy facilitates decision on control strategies.This study aimed to assess the prevalence of $P$. falciparum malaria infection and possible associated risk factors in pregnant women attending antenatal clinic in the periurban area of Bobo-Dioulasso.

\section{Methods}

\section{Study site and study design}

A cross sectional study was performed from September to December 2010 in Lafiabougou and Kua two primary health facilities both located in the periurban area of Bobo-Dioulasso (Burkina Faso). In this area, the high transmission season of malaria lasts 4 months from August to November. The entomological inoculation rate (EIR) was about 63 infective bites per habitant per year "unpublished observations".

\section{Data collection}

All pregnant women presenting for their routine antenatal clinic visits were included in the study after explaining to them the purpose of the study and signing informed consent. Each participant was evaluated only once. Data were obtained through a questionnaire and included the following information: social-demographic profile, malaria history, obstetric history including parity, gestational age. Gestational age was calculated from the first day of bleeding of the last menstrual period. Only two pregnant women refused to participate to the study because their husband did not allow them to participate to the study.

\section{Laboratory methods}

Blood samples were taken by finger prick for checking both malaria parasite and hemoglobin concentration. Blood smear and thick drop assays were stained with
10\% Giemsa dye. Smears were fixed with May Grunvald for three minutes before staining with Giemsa for 20 minutes. Parasite density was determined by counting asexual forms of the parasite per 200 leukocytes assuming 8,000 leukocytes/ $\mu \mathrm{L}$ of blood. A slide was considered negative if no parasite was found after counting 500 leukocytes. All the slides were double-checked blindly and for discrepant results a third consensus reading was performed.

Hemoglobin concentration was determined by the hematic acid method using a hemoglobinometer (HemoCue $\mathrm{AB}$, Angelhom, Sweden) and were classified as anemia $(<11 \mathrm{~g} / \mathrm{dL})$, severe anemia $(<8 \mathrm{~g} / \mathrm{dL})$ and normal $(\geq 11 \mathrm{~g} / \mathrm{dL})[10]$.

\section{Sample size}

The sample size calculation was based on an assumed prevalence of malaria in pregnant women attending antenatal clinic and benefiting from IPT-SP of 13.8\% [5], a precision of $5 \%$ and for a significant result at the $5 \%$ level. A sample of 193 pregnant women was needed. Then we sampled 193 pregnant women in each trimester of pregnancy resulting a total size of 579 pregnant women.

\section{Statistical analysis of data}

Data were double entered in EpiData 3.1 software and analyzed by using Stata 12 software.

The prevalence of $P$. falciparum malaria infection has been estimated with a 95\% confidence interval (CI). Univariate analyses were performed by using the Pearson Chi-square or Fisher's exact tests to compare proportions for categorical variables. Comparison between continuous variables with normal distributions including age and hemoglobin concentration was done by the Student's t-test or the Anova test. The Wilcoxon rank sum test and the Kruskall-Wallis test were used to compare continuous variables with non-normal distributions (parasite density and number of ANC visits).

Simple and multiple logistic regression models were also used as described below. The variable malaria (including with and without $P$. falciparum malaria infection categories) was considered as the dependent variable. The independent variables included age, schooling, gestational age, parity, use of IPTp-SP and insecticide-treated net (ITN) during pregnancy, number of ANC visits and anaemia. Variables were categorized as follows: age ( $<20$ years old, $\geq 20$ years old); parity as primigravidae, secundigravidae, multigravidae $(\geq 3)$; gestational age [first trimester $(<14$ weeks), second trimester $(14-27$ weeks) and third trimester ( $\geq 28$ weeks)], number of ANC visits $(0-1,2$ and $\geq 3)$. To investigate the association between the several independent variables and malaria, we began by performing simple logistic regressions with each independent variable. Next, we applied multiple regression 
models to control possible confusion. Variables exhibiting statistically significant associations $(\mathrm{p}<0.05)$ or with important epidemiological meanings were included. These co-variables were kept in models, independent of their significance, in univariate analysis due to their possible relevance in the final results; thus, we could analyze their possible influence when considered together with the other variables.

\section{Ethical considerations}

The study was initially discussed with health authorities and community leaders to obtain their assent. This study was approved by the National Ethics Committee for health research, Ouagadougou Burkina Faso (number 2010-054). A written informed consent was obtained from all pregnant prior to their enrolment in the study. For illiterate pregnant women, the informed consent discussion process was witnessed by an impartial individual. In those cases, the informed consent form had been signed with a thumbprint.

All women with anaemia or positive for malaria have been treated orally with folic acid plus ferrous and quinine $300 \mathrm{mg}$ (24 mg/day until 7 days), respectively.

\section{Results}

\section{Baseline characteristics of pregnant women attending antenatal clinic}

The baseline characteristics of the 579 pregnant women involved in the study are summarized in Table 1. Briefly, the pregnant women were young $(24.7$ years \pm 5.7$)$ and most of them had no formal education (61.1\%), and were multigravidae (44.7\%). Moreover $46.9 \%$ and $26.7 \%$ of pregnant women benefited from ITN and at least two doses of IPTp-SP, respectively. The proportion of pregnant women receiving the recommended 2 or 3 doses of IPTp-SP was higher during the 3rd trimester $(\mathrm{p}<0.001)$. Of the 579 participating women, $50.4 \%$ reported attending ANC at least once during their pregnancy. The number of ANC visits varied from 0 to 2 with a median of 2 visits. Among ANC attendees, majority (39.9\%) made their first visit during the second trimester. Only $2.8 \%$ of the ANC attendees had complete attendance (considered to be at least four ANC visits during pregnancy). The prevalence of $P$. falciparum parasitaemia was $18.1 \%(95 \% \mathrm{CI}=[15.1-21.2])$ and the geometric mean of the parasite density (GMPD) was 2254 parasites/ $\mu \mathrm{L}$ $(95 \% \mathrm{CI}=[1755-2894])$. The geometric mean of the density of $P$. falciparum parasitaemia was significantly lower in pregnant women who had used IPTp-SP $(\mathrm{P}<0.001)$. The haemoglobin concentration mean was significantly lower in pregnant women with malaria infection $(9.8 \mathrm{~g} / \mathrm{dL} \pm 1.6)$ than in those who had no malaria infection $(10.6 \mathrm{~g} / \mathrm{dL} \pm 1.7)(\mathrm{P}<0.001)$.

\section{IPTp-SP uptake at ANC clinic}

The proportion of women receiving the recommended 2 or 3 doses of IPTp-SP increased $(\mathrm{p}<0.001)$ with the number of ANC visits attended (Table 2).

\section{Factors associated with malaria among pregnant women attending antenatal clinic}

Multivariate analysis indicated that, lower level of education (AOR 1.9, 95\% $\mathrm{CI}=[1.2-3.2]$ ), parity [primigravidae (AOR 5.0, 95\% $\mathrm{CI}=[2.5-9.8]$ ) and secundigravidae (AOR $2.1,95 \% \mathrm{CI}=[1.2-3.8])]$, and anaemia (AOR 2.1, 95\% CI = [1.3-3.5]) were significantly associated with $P$. falciparum malaria infection. The use of IPTp-SP was not associated with $P$. falciparum malaria infection (Table 3 ).

\section{Discussion}

This study aimed to assess the prevalence of P. falciparum malaria infection and possible associated risk factors in pregnant women attending ANC in the periurban area of Bobo-Dioulasso. The prevalence of maternal peripheral $P$. falciparum infection assessed by microscopy was $24 \%$ in urban area [12] and varied from $19.4 \%$ to $50.8 \%$ in rural area $[5-9,13]$. An additional file shows this in more detail [see Additional file 1]. The higher observed rates in rural area could be explained by the high transmission level of malaria in rural area of Burkina Faso (unpublished observations). The difference between our results with those reported in Ouagadougou, the capital of Burkina Faso [12] could be due to the lower use of IPTp-SP in that study. Our rate was higher compared to that found in the rural area of Ghana in 2012 [24]. Indeed, all pregnant women included in the Ghanaian study had benefited from at least 2 doses IPTp-SP. A prevalence of $10.9 \%$ was found in Luanda, Angola, an urban site with similar malaria epidemiological characteristics [25] even though the survey had been conducted during high the transmission season of malaria. Our findings about the prevalence of malaria may be underestimated due to the use of microscopy of Giemsa-stained blood smears of peripheral blood for diagnosis. The use of PCR could have improved the sensitivity and specificity of the diagnosis as previously reported in Burkina Faso [13] nevertheless the high microscopic prevalence is of serious concern.

This study showed that lack of formal education was a risk factor for $P$. falciparum malaria.This agrees with previous findings in India [26] and highlights the need of more sensitization to increase the use of malaria prevention measures. Parity was associated with malaria infection even adjusted for age as previously shown [5]. Association between the decrease in risk of malaria infection and parity has been reported in several studies $[27,28]$. However, in none of these studies, the association between parity and malaria infection was adjusted for women's age. A common explanation of the association 
Table 1 Baseline characteristics of pregnant women attending ANC in Bobo-Dioulasso

\begin{tabular}{|c|c|c|c|c|c|}
\hline Characteristics & $\begin{array}{l}\text { Total } \\
(n=579)\end{array}$ & $\begin{array}{l}\text { 1st } \\
\text { Trimester }(n=193)\end{array}$ & $\begin{array}{l}\text { 2nd } \\
\text { Trimester }(n=193)\end{array}$ & $\begin{array}{l}\text { 3rd } \\
\text { Trimester }(n=193)\end{array}$ & $P$ \\
\hline Mean age (year), & $24.7 \pm 5.7$ & $24.5 \pm 5.7$ & $24.2 \pm 5.4$ & $25.4 \pm 5.8$ & $0.09^{\mathrm{a}}$ \\
\hline \multicolumn{6}{|l|}{$\pm \mathrm{SD}$} \\
\hline$<20$ & 108 (18.6) & $41(21.2)$ & $39(20.2)$ & $28(14.5)$ & \multirow[t]{2}{*}{$0.2^{p}$} \\
\hline$\geq 20$ & $471(81.4)$ & $152(78.8)$ & $154(79.8)$ & $165(85.5)$ & \\
\hline \multicolumn{5}{|l|}{ Education } & \multirow[t]{3}{*}{$0.5^{p}$} \\
\hline No formal schooling & $353(61.1)$ & $113(58.5)$ & $124(64.6)$ & $116(60.1)$ & \\
\hline Formal schooling & $225(38.9)$ & $80(41.5)$ & $68(35.4)$ & $77(39.9)$ & \\
\hline \multicolumn{5}{|l|}{ Parity } & \multirow[t]{4}{*}{$0.01^{P}$} \\
\hline Primigravidae & $170(29.4)$ & $72(37.3)$ & $61(31.6)$ & 37 (19.1) & \\
\hline Secundigravidae & $150(25.9)$ & $43(22.3)$ & $48(24.9)$ & 59 (37.6) & \\
\hline Multigravidae & $259(44.7)$ & $78(40.4)$ & $84(43.5)$ & $97(50.3)$ & \\
\hline ITN Use or ownership & $271(46.9)$ & $90(46.9)$ & $91(47.2)$ & $90(46.6)$ & $0.9^{p}$ \\
\hline \multicolumn{6}{|l|}{ ANC visits } \\
\hline Median, quartiles & $2(0-2)$ & - & $1(1-2)$ & $2(1-3)$ & \multirow[t]{2}{*}{$<0.001^{\mathrm{w}}$} \\
\hline No prior ANC visit & $287(49.6)$ & $190(98.5)$ & $88(45.6)$ & $9(4.7)$ & \\
\hline 1 & $125(21.6)$ & $3(1.5)$ & 77 (39.9) & $45(23.3)$ & \multirow[t]{3}{*}{$<0.001^{f}$} \\
\hline 2 & 108 (18.6) & $0(0.0)$ & $24(12.4)$ & $84(43.5)$ & \\
\hline$\geq 3$ & $59(10.2)$ & $0(0.0)$ & $4(2.1)$ & $55(28.5)$ & \\
\hline \multicolumn{6}{|l|}{ Doses of IPTp-SP } \\
\hline 0 & $352(60.8)$ & $193(100.0)$ & $139(72.0)$ & $20(10.4)$ & \multirow[t]{3}{*}{$<0.001^{f}$} \\
\hline 1 & $124(21.4)$ & $0(0.0)$ & $46(23.8)$ & $78(40.4)$ & \\
\hline$\geq 2$ & $103(17.8)$ & $0(0.0)$ & $8(4.2)$ & $95(49.2)$ & \\
\hline Peripheral parasitaemia & $105(18.1)$ & $46(23.8)$ & $37(19.2)$ & $22(11.4)$ & $0.006^{p}$ \\
\hline GMPD/ $\mu \mathrm{L}$, & 2254 & 2300 & 1886 & 2913 & \multirow[t]{2}{*}{$0.3^{k}$} \\
\hline$[95 \%$ IC $]$ & [1755-2894] & [1600-3008] & [1253-2839] & {$[1475-5757]$} & \\
\hline Mean haemoglobin $(\mathrm{g} / \mathrm{dL}), \pm \mathrm{SD}$ & $10.5 \pm 1.7$ & $11.0 \pm 1.7$ & $10.1 \pm 1.6$ & $10.3 \pm 1.7$ & $<0.001^{\mathrm{a}}$ \\
\hline Anaemia (\% $\mathrm{Hb}<11 \mathrm{~g} / \mathrm{dL})$ & $346(60.0)$ & $90(46.9)$ & $132(68.4)$ & $124(64.6)$ & $<0.001^{\mathrm{P}}$ \\
\hline Moderate to severe anaemia $(\% \mathrm{Hb}<8 \mathrm{~g} / \mathrm{dL})$ & $37(6.4)$ & $5(2.6)$ & $17(8.8)$ & $15(7.8)$ & $0.03^{\mathrm{P}}$ \\
\hline
\end{tabular}

${ }^{\mathrm{a}}$ Determined by Anova Test. ${ }^{\mathrm{P}}$ Determined by Pearson Chi-square Test.

${ }^{f}$ Comparison between 2 nd and 3rd trimester determined by Chi-square (Fischer exact) Test.

${ }^{\mathrm{w}}$ Determined by Wilcoxon rank sum Test. ${ }^{\mathrm{k}}$ Determined by Kruskal-Wallis Test.

Table 2 Distribution of IPTp-SP doses according to the number of ANC visits

\begin{tabular}{lllll}
\hline Characteristics & \multicolumn{2}{l}{ Number of ANC visits } & P \\
\cline { 2 - 3 } & $\mathbf{0 - 1}$ & $\mathbf{2}$ & $\geq \mathbf{3}$ & \\
\hline IPTp-SP doses & & $1(0.9)$ & $0(0.0)$ & $<0.001^{f}$ \\
0 & $351(85.2)$ & $53(49.1)$ & $12(20.3)$ & \\
1 & $59(14.3)$ & $54(50.0)$ & $47(79.7)$ & \\
$\geq 2$ & $2(0.5)$ & & & \\
\hline
\end{tabular}

fDetermined by Chi-square (Fischer exact) Test. between malaria infection and parity is that pregnancy is associated with a decrease in immunity [2]. In Mali, parity was significantly associated with malaria infection only when the analysis was not adjusted for age [21]. Other authors found no association between parity and malaria infection $[19,23,25]$. In the present study and as well as in that of Adam et al. [23], age was not significantly associated with malaria infection in contrast to the previous observations $[19,21,22]$. Previous sulfadoxine-pyrimethamine chemoprophylaxis was not associated with the prevalence of malaria infection when adjusted for different factors. In addition the coverage of the recommended 2 or 3 IPTp-SP among the 3rd trimester women was very low $(49.2 \%)$ but it is higher than the recent reported coverage (10-19\%) for Burkina Faso [29]. 
Table 3 Risk factors associated with malaria among pregnant women attending ANC in Bobo-Dioulasso

\begin{tabular}{|c|c|c|c|c|}
\hline Potential factors & Malaria & $\begin{array}{l}\text { Crude OR } \\
{[95 \% \text { IC] }}\end{array}$ & $\begin{array}{l}\text { Adjusted OR } \\
{[95 \% \text { IC] }}\end{array}$ & $\mathbf{P}$ \\
\hline \multicolumn{5}{|l|}{ Age } \\
\hline$\geq 20$ years & $17.0(80 / 471)$ & 1 & 1 & - \\
\hline$<20$ years & $23.1(25 / 108)$ & $1.5[0.9-2.4]^{*}$ & $1.6[0.8-3.2]$ & 0.2 \\
\hline \multicolumn{5}{|l|}{ Education } \\
\hline Formal schooling & $19.8(70 / 353)$ & 1 & 1 & - \\
\hline No formal schooling & $15.6(35 / 225)$ & $1.3[0.9-2.1]^{*}$ & $1.9[1.2-3.2]$ & 0.01 \\
\hline \multicolumn{5}{|l|}{ Gestational age } \\
\hline 3rd trimester & $11.4(22 / 193)$ & 1 & 1 & - \\
\hline 2nd trimester & $19.7(37 / 193)$ & $1.8[1.04-3.3]$ & $0.7[0.3-1.5]$ & 0.4 \\
\hline 1st trimester & $23.8(46 / 193)$ & 2.4 [1.4-4.23] & $0.8[0.3-1.9]$ & 0.6 \\
\hline \multicolumn{5}{|l|}{ Parity } \\
\hline Multigravidae & $11.2(29 / 259)$ & 1 & 1 & 1 \\
\hline Secundigravidae & $18.0(27 / 150)$ & $1.7[0.9-3.1]$ & $2.1[1.2-3.8]$ & 0.02 \\
\hline Primigravidae & $28.8(49 / 170)$ & $3.2[1.9-5.3]$ & $5.0[2.5-9.8]$ & $<0.001$ \\
\hline \multicolumn{5}{|l|}{ ANC visits } \\
\hline $0-1$ & $22.3(92 / 412)$ & 1 & 1 & 1 \\
\hline 2 & $4.6(5 / 108)$ & $0.2[0.1-0.4]$ & $0.3[0.1-1.0]$ & 0.05 \\
\hline$\geq 3$ & $13.6(8 / 59)$ & $0.5[0.2-1.2]$ & $1.3[0.3-5.2]$ & 0.7 \\
\hline \multicolumn{5}{|l|}{ IPTp-SP dose } \\
\hline 0 & $23.4(83 / 352)$ & 1 & 1 & - \\
\hline 1 & $11.3(14 / 124)$ & $0.4[0.2-0.8]$ & $0.5[0.2-1.2]$ & 0.1 \\
\hline$\geq 2$ & $7.8(8 / 103)$ & $0.3[0.1-0.6]$ & $0.3[0.1-1.5]$ & 0.1 \\
\hline \multicolumn{5}{|l|}{ ITN } \\
\hline No & $20.9(64 / 307)$ & 1 & 1 & 1 \\
\hline Yes & $14.8(40 / 271)$ & $0.7[0.4-1.0]$ & $0.8[0.5-1.5]$ & 0.4 \\
\hline \multicolumn{5}{|l|}{ Anaemia } \\
\hline No & $12.5(29 / 231)$ & 1 & 1 & 1 \\
\hline Yes & $21.7(76 / 346)$ & $2.0[1.2-3.1]$ & $2.1[1.3-3.5]$ & 0.003 \\
\hline
\end{tabular}

*P value $=0.2$.

Furthermore only $46.6 \%$ of them reported ITN ownership. All these findings together highlight the WHO 2004 malaria prevention program failure and suggest the need to replace SP. However is it worth replacing SP when the WHO goal of four ANC visits [3] during pregnancy is not yet achieved? Indeed, the majority of the 3rd trimester women (71.5\%) have attended less than three ANC visits. This is of serious concern because the cost of an ANC consultation is officially free of charge in Burkina Faso. The lower level of education of pregnant women could have contributed to the low level of correct ANC attendance. Thus educating pregnant women on focused ANC visits is recommended. Moreover the whole health system needs to be revitalized including antenatal care staffs that also need to be educated and enthused so that they can explain to women to try to meet the number of visits; the government needs to support access to antenatal care in rural areas.

The coverage of the recommended SP dosage increased with the number of ANC visits as previously reported in Benin [30] and in Cameroon [31]. However in Gabon, almost $40 \%$ of delivering women with more than three ANC visits had no or partial SP uptake [32].

This suggests that the number of ANC visits does not necessarily ensure the complete IPTp- SP coverage. Indeed, it has been shown that the coverage of IPTpSP increases and ANC attendance is substantial when SP is supervised [32]. Thus further studies are needed to explore barriers to complete IPTp-SP coverage in Bobo-Dioulasso.

The mean haemoglobin was significantly lower in women with malaria infection. This agrees with findings 
not only in areas of stable transmission of malaria [25,33] but also in areas with unstable transmission [23,34]. Anaemia was the main complication of malaria infection found in our study and remains the most frequent consequence of malaria during pregnancy irrespective of transmission level and pre-pregnancy level of malaria immunity [35].

\section{Conclusion}

Our results suggest that $P$. falciparum is common in pregnant women attending antenatal clinic in Bobo-Dioulasso and that anemia is an important complication associated with $P$. falciparum infection. Lack of formal education and parity even adjusted for age are main risk factors for malaria. The use of IPTp-SP was not associated with P. falciparum malaria infection.

\section{Additional file}

Additional file 1: Prevalence of maternal peripheral $P$. falciparum infection from 2004 to 2011 in Burkina Faso. The data provided show the prevalence of maternal peripheral $P$. falciparum infection reported in Burkina Faso, organized by type of study, period of study, location, pregnant women's data including age and parity.

\section{Abbreviations}

ANC: Antenatal clinic; AOR: Adjusted odd ratio; Cl: Confident interval; Dhfr: Dihydrofolate reductase; dL: Deciliter; g: Gramme; GMPD: Geometric mean of the parasite density; Hb: Hemoglobin; ITP-SP: Intermittent preventive treatment with sulfadoxine pyrimethamine; ITN: Insecticide-Treated Net; OR: Odd ratio; WHO: World Health Organization.

\section{Competing interests}

The authors declare that they no competing interests.

\section{Authors' contributions}

MC conceived, designed and implemented the study and carried out the microscopic analysis. RTG was involved in the study design and supervised the implementation of the study. MC drafted the manuscript performed statistical analysis. IS, SB, GL, DB and RTG helped to critically analysed the manuscript. All authors read and approved the final version of the manuscript.

\section{Acknowledgements}

We wish to thank all the pregnant women for their excellent cooperation and we are very grateful to both Lafiabougou and Kua primary health facilities staffs. Thanks are also extended to the "Projet Interuniversitaire Ciblé, Institut Supérieur des sciences de la santé" (PIC/INSSA) for the financial support of the study.

\section{Author details}

${ }^{1}$ Centre MURAZ, Bobo-Dioulasso, Burkina Faso. ${ }^{2}$ Centre Hospitalier Universitaire Souro Sanou, Bobo-Dioulasso, Burkina Faso. ${ }^{3}$ Institut Supérieur des sciences de la Santé, Université Polytechnique de Bobo-Dioulasso, Bobo-Dioulasso, Burkina Faso. ${ }^{4}$ District sanitaire de Dafra, Bobo-Dioulasso, Burkina Faso.

Received: 28 April 2014 Accepted: 11 November 2014 Published online: 19 November 2014

\section{References}

1. Steketee RW, Nahlen BL, Parise MEMC: The burden of malaria in pregnancy in malaria-endemic areas. Am J Trop Med Hyg 2001, 64:28-35.

2. Brabin BJ: An analysis of malaria in pregnancy in Africa. Bull World Health Organ 1983, 61:1005-1016.
3. WHO: A Strategic Framework for Malaria Prevention and Control During Pregnancy in the African Region. In World Health Organization Regional Office for Africa, AFR/MAL/04/01. 2004.

4. Ministère de la santé Burkina Faso: Annuaire Statistique 2011. Ouagadougou: 2012.

5. Sirima SB, Cotte AH, Konaté A, Moran AC, Asamoa K, Bougouma EC, Diarra A, Ouédraogo A, Parise ME, Newman RD: Malaria prevention during pregnancy: Assessing the disease burden one year after implementing a program of intermittent preventive treatment in Koupela district, Burkina Faso. Am J Trop Med Hyg 2006, 75(2):205-211.

6. Gies S, Coulibaly SO, Ouattara FT, Ky C, Brabin BJ, D'Alessandro U: A community effectiveness trial of strategies promoting intermittent preventive treatment with sulphadoxine-pyrimethamine in pregnant women in rural Burkina Faso. Malar J 2008, 7:180.

7. Gies S, Coulibaly SO, Ouattara FT, D'Alessandro U: Individual efficacy of intermittent preventive treatment with sulfadoxine-pyrimethamine in primi- and secundigravidae in rural Burkina Faso: impact on parasitaemia, anaemia and birth weight. Trop Med Int Health 2009, 14(2):174-182.

8. Ouédraogo A, Bougouma EC, Diarra A, Konaté A, Nébié I, Tiono B, Sirima SB: Comparative impact of three malaria preventive regimens during pregnancy on maternal anemia due to malaria in Burkina Faso. Med Mal Infect 2008, 38(4):180-186.

9. Tiono AB, Ouedraogo A, Bougouma EC, Diarra A, Konaté AT, Nébié I, Sirima SB: Placental malaria and low birth weight in pregnant women living in a rural area of Burkina Faso following the use of three preventive treatment regimens. Malar J 2009, 8:224.

10. Valea I, Tinto H, Drabo MK, Huybregts L, Henry M, Roberfroid D, Guiguemde RT, Kolsteren P, Alessandro UD: Intermittent preventive treatment of malaria with sulphadoxine-pyrimethamine during pregnancy in Burkina Faso: effect of adding a third dose to the standard two-dose regimen on low birth weight, anaemia and pregnancy outcomes. Malar J 2010, 9(1):324.

11. Valea I, Tinto H, Drabo MK, Huybregts L, Sorgho H, Ouedraogo J, Guiguemde RT, Geertruyden JP, Kolsteren P, Alessandro UD: An analysis of timing and frequency of malaria infection during pregnancy in relation to the risk of low birth weight, anaemia and perinatal mortality in Burkina Faso. Malar J 2012, 11:71

12. Douamba Z, Bisseye C, Djigma FW, Compaore TR, Bazie T, Pietra V, Nikiema $J \mathrm{~B}$, Simpore J: Asymptomatic malaria correlates with anaemia in pregnant women at Ouagadougou, Burkina Faso. J Biomed Biotechnol 2012, 2012:198317.

13. Kattenberg JH, Tahita CM, Versteeg IAJ, Tinto H, Traore M, Alessandro UD, Schallig HD MP, Mens PF: Evaluation of antigen detection tests, microscopy, and polymerase chain reaction for diagnosis of malaria in peripheral blood in asymptomatic pregnant women in Nanoro, Burkina Faso. Am J Trop Med Hyg 2012, 87(2):251-256.

14. Kattenberg JH, Tahita CM, Versteeg I, Tinto H, Traore Coulibaly M, Schallig HD MP: Antigen persistence of rapid diagnostic tests in pregnant women in Nanoro, Burkina Faso and the implications for the diagnosis of malaria in pregnancy. Trop Med Int Health 2012, 17:550-557.

15. Bamba S, Séré A, Nikiéma R, Halidou T, Thiéba B, Dao B, Guiguemdé RT: Intermittent preventive treatment with sulfadoxine-pyrimethamine for malaria in pregnant women: efficacy and compliance in two urban hospitals in Burkina Faso. Pan Afr Med J 2013, 14:105.

16. Coulibaly SO, Kayentao K, Taylor S, Guirou E, Khairallah C, Guindo N, Djimde M, Bationo R, Soulama A, Dabira E, Barry B, Niangaly M, Diakite H, Konate S, Keita M, Traore B, Meshnick SR, Magnussen P, Doumbo OK, ter Kuile FO: Parasite clearance following treatment with sulphadoxinepyrimethamine for intermittent preventive treatment in Burkina-Faso and Mali: 42-day in vivo follow-up study. Malar J 2014, 13:41.

17. Miaffo C, Some F, Kouyate B, Jahn AMO: Malaria and anemia prevention in pregnant women of rural Burkina Faso. BMC Pregnancy Childbirth 2004, 4(1):18.

18. Tako EA, Zhou A, Lohoue J, Leke R, Taylor DW, Leke RFG: Risk factors for placental malaria and its effect on pregnancy outcome in Yaounde, Cameroon. Am J Trop Med Hyg 2005, 72(3):236-242.

19. Rogerson SJ, Broek NR, Vanden, Chaluluka E, Qongwane C, Mhango CG, Molyneux ME: Malaria and anemia in antenatal women in Blantyre, Malawi: a twelve-month survey. Am J Trop Med Hyg 2000, 62(3):335-340.

20. Saute F, Menendez C, Mayor A, Aponte J, Gomez-olive X, Dgedge M, Alonso P: Malaria in pregnancy in rural Mozambique: the role of parity, submicroscopic and multiple Plasmodium falciparum infections. Trop Med Int Health 2002, 7(1):19-28 
21. Dicko A, Mantel C, Aly M, Doumbia S: Risk factors for malaria infection and anemia for pregnant women in the Sahel area of Bandiagara. Mali Acta Trop 2003, 89:17-23.

22. Bouyou-akotet MK, lonete-collard DE, Mabika M, Kendjo E, Matsiegui PB, Mavoungou E, Kombila M: Prevalence of Plasmodium falciparum infection in pregnant women in Gabon. Malar J 2003, 7:1-7.

23. Adam I, Khamis AH, Elbashir MI: Prevalence and risk factors for Plasmodium falciparum malaria in pregnant women of eastern Sudan. Malar J 2005, 4:4-7.

24. Osei Tutu E, Lawson B, Browne E: The effectiveness and perception of the use of sulphadoxine-pyrimethamine in intermittent preventive treatment of malaria in pregnancy programme in Offinso District of Ashanti Region, Ghana. Malar J 2011, 10:385.

25. Campos PA, Valente B, Campos RB, Gonçalves L, Rosário VE: Plasmodium falciparum infection in pregnant women attending antenatal care in Luanda, Angola. Rev Soc Bras Med Trop 2012, 45(3):369-374.

26. Singh N, Singh MP, Wylie BJ, Hussain M, Kojo YA, Shekhar C, Sabin L, Desai M: Malaria prevalence among pregnant women in two districts with differing endemicity in Chhattisgarh, India Neeru. Malar J 2012, 11:274.

27. Fleming AF: Tropical obstetrics and gynecology. Anemia in pregnancy in Tropical Africa. Trans R Soc Trop Med Hyg 1989, 83:441-448.

28. Matteelli A, Donato F, Shein A: Malaria and anemia in pregnant women in urban Zanzibar, Tanzania. Ann Trop Med Parasitol 1994, 88(5):475-483.

29. Van Eijk AM, Hill J, Larsen DA, Webster J, Steketee RW, Eisele TP, Kuile FO: Coverage of intermittent preventive treatment and insecticide-treated nets for the control of malaria during pregnancy in sub-Saharan Africa: a synthesis and meta-analysis of national survey data, 2009-11. Lancet Infect Dis 2013, 13(12):1029-1042.

30. D'Almeida TC, Agboton-Zoumenou M-A, Garcia A, Massougbodji A, Briand $V$, Imorou Y, Cottrell G: Field evaluation of the intermittent preventive treatment of malaria during pregnancy (IPTp) in Benin: evolution of the coverage rate since its implementation. Parasit Vectors 2011, 4:108.

31. Anchang-kimbi JK, Achidi EA, Apinjoh TO, Mugri RN, Chi HF, Tata RB, Nkegoum B, Mendimi JN, Sverremark-ekström E, Troye-blomberg M: Antenatal care visit attendance, intermittent preventive treatment during pregnancy (IPTp) and malaria parasitaemia at delivery. Malar J 2014, 13:162.

32. Bouyou-Akotet MK, Mawili-Mboumba DP, Kombila M: Antenatal care visit attendance, intermittent preventive treatment and bed net use during pregnancy in Gabon. BMC Pregnancy childbirth 2013, 13:52.

33. Menendez C, Fleming AF, Alonso PL: Malaria-related anaemia. Parasitol Today 2000, 16:469-476.

34. Newman RD, Hailemariam A, Jimma D, Degifie A, Kebede D, Rietveld AEC, Nahlen BL, Barnwell JW, Steketee RW: Burden of malaria during pregnancy in areas of stable and unstable transmission in Ethiopia during a nonepidemic year. J Infect Dis 2003, 187(11):1765-1772.

35. Menendez C: Malaria during pregnancy: A priority area of malaria research and control. Parasitol Today 1995, 11:178-183.

doi:10.1186/s12879-014-0631-z

Cite this article as: Cisse et al:: Prevalence and risk factors for Plasmodium falciparum malaria in pregnant women attending antenatal clinic in Bobo-Dioulasso (Burkina Faso). BMC Infectious Diseases 2014 14:631.

\section{Submit your next manuscript to BioMed Central and take full advantage of:}

- Convenient online submission

- Thorough peer review

- No space constraints or color figure charges

- Immediate publication on acceptance

- Inclusion in PubMed, CAS, Scopus and Google Scholar

- Research which is freely available for redistribution

Submit your manuscript at www.biomedcentral.com/submit
C Biomed Central 